\title{
The Neuropathology of "Typical”' Friedreich's Ataxia in Quebec
}

\author{
J.B. Lamarche, B. Lemieux, H.B. Lieu
}

\begin{abstract}
We present the pathological data from the autopsies performed on 6 Friedreich's disease patients since the start of the Quebec Cooperative Study. All patients met the strict diagnostic criteria of the QCSFA. The anatomical lesions found in the peripheral and central nervous system were similar in all 6 cases and do not differ from those described in the literature. The clinical findings correlate closely with the histological lesions found in the peripheral nervous system and spinal cord. The evidence of segmental demyelination and remyelination in the spinal ganglia and posterior roots further supports the dying-back axonopathy hypothesis.
\end{abstract}

RÉSUMÉ: Nous décrivons les données pathologiques provenant des autopsies des 6 patients avec maladie de Friedreich décédés depuis le début de l'Etude Coopérative. Tous les patients remplissaient les critères de diagnostic stricts du QCSFA. Les lésions anatomiques trouvées dans le système nerveux périphérique et central étaient semblables chez les 6 patients et ne diffèrent pas de celles rapportées dans la littérature. Les signes et symptômes cliniques sont en bonne corrélation avec les lésions histologiques trouvées dans le système nerveux périphérique et la moelle épinière. L'évidence de démyélination et de remyélination segmentaire mise en lumière dans le ganglion spinal et les racines postérieures contribue à appuyer l'hypothèse d'une axonopathie de type "dying-back".

Can. J. Neurol. Sci. 1984; 11:592-600

Since the formation of the Quebec Cooperative group for the study of Friedreich's ataxia, post-mortem material from six ataxic patients was examined. All these patients suffered from "typical" Friedreich's ataxia (Geoffroy et al., 1976) and died after similar clinical courses ranging from 6 to 12 years. They came from 5 families, in which there was close consanguinity in one.

The purpose of this paper is to report the detailed neuropathological findings in these patients and to correlate the morphological data with the clinical picture and the course of the disease. All cases were essentially similar as to age of onset, major clinical findings and clinical course. Whatever the duration of the disease, the lesions of the peripheral nervous system and of the spinal cord were uniform and conform to the known description of classical Friedreich's ataxia. Brain stem lesions although variable from case to case, did not differ from those reported in the literature and did not fully correlate with the clinical findings. The presence of axonal swellings consistently found in the cerebellum suggests a degeneration of the Purkinje cells in Friedreich's ataxia.

\section{Patients ANd Methods}

A complete post-mortem examination was performed in most cases. In cases $1,2,4$, and 6 the entire brain was cut sagitally in half and one hemisphere was immediately frozen for preservation in a tissue bank. The other half was fixed in $10 \%$ neutral buffered formalin and after coronal sections representative samples were embedded in paraffin and stained with hematoxyline and eosin, Bodian for axones, Luxol fast blue for myelin and Holzer for glial fibers. The spinal cord was also removed and sections were obtained from cervical, thoracic and lumbosacral levels together with corresponding segments of anterior and posterior roots and spinal ganglia. Samples from the peripheral nervous system including autonomic nerves and ganglia were also obtained.

In cases 3 and 5 , the patients died in other institutions and the pathological material, whole brain and segments of spinal cord fixed in $10 \%$ formalin, was sent to us.

In 2 cases, segments of peripheral nerve and roots were fixed in a mixture of $2 \%$ glutaraldehyde and $2 \%$ paraformaldehyde in phosphate buffer and embedded in Epon. Semi-thin sections were stained with toluidine blue. The abbreviated pedigrees of the families are shown in figures I to V. Inheritance is autosomal recessive.

The pathological material was first studied at the time of the autopsy and was re-examined before the preparation of the results by the same observer (JBL). No significant differences were recorded between the initial histological examination and 


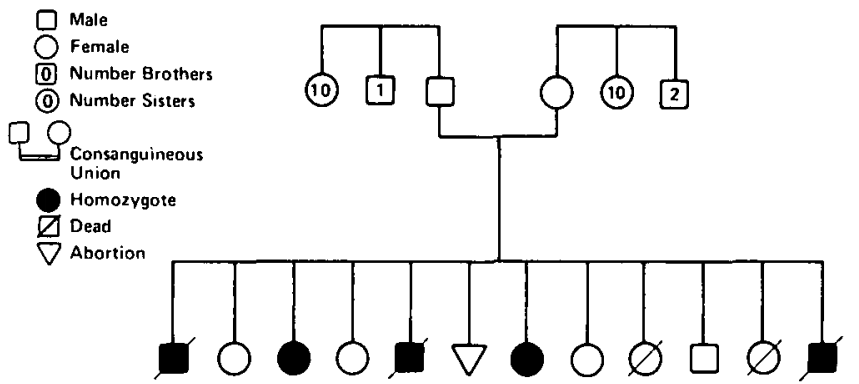

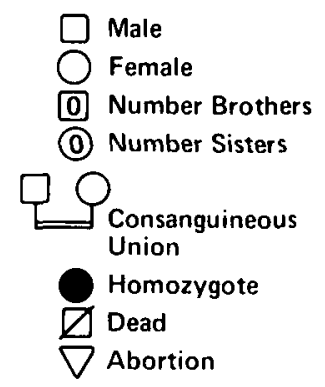

11
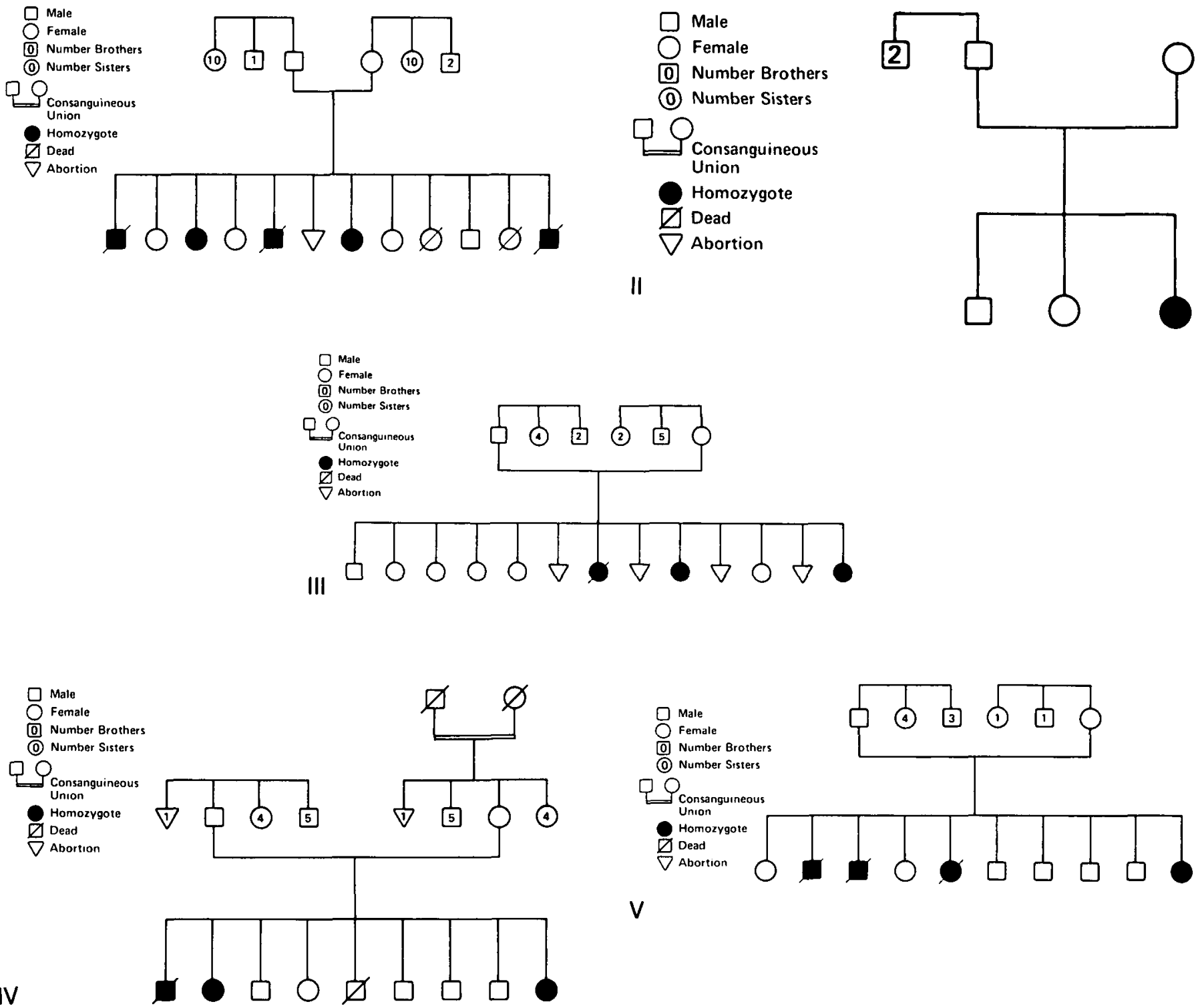

FAMILY TREES

the review. Any alterations which were found doubtful were not retained.

\section{CaSe 1 (L.F. - A-58-77) (Family Tree III)}

\section{Clinical Summary:}

This patient was a 6 pound 8 ounce product of an uncomplicated term pregnancy, and a vaginal delivery without complication after breech presentation. Early growth and development were normal and she walked at 9 months of age. Gait disturbances were first manifest at the age of 2 years. She became confined to a wheel chair at the age of 10 . Cardiac manifestations were reported (case 1, Lamarche et al., 1980). Neurological evaluations was carried out at the age of 15 and reported as S.B.L.-3 in the group 1 Friedreich's ataxia (Geoffroy et al., 1976). At that time, there was a marked right dorsal scoliosis which was found to be at $45^{\circ}$ on X-rays. Dysarthria which began at the age of 8 was moderate. Pes cavus was present and a mild nystagmus was noted. No deafness or clinical diabetes was observed. The EEG was normal. She died at age 18 after a brief episode of nausea, vomiting of blood stained material and progressively prolonged apneic spells.

An autopsy was carried out 6 hours after her death.

\section{Summary of General Autopsy Findings:}

Pertinent findings outside the nervous system included bilateral pes cavus, marked scoliosis, generalized muscular atrophy affecting particularly the lower limbs and hypertrophic cardiomyopathy.

There was chronic passive congestion of the liver with erosive gastrooesophagitis and blood in the digestive tract. In the lungs, there were multiple peripheral thrombo-emboli associated with hemorragic infarcts.

\section{Neuropathology:}

\section{a) Gross findings}

The brain weighed $1360 \mathrm{~g}$ in the fresh state. The brain was within normal limits on external examination and after coronal sections. The spinal cord and posterior roots appeared atrophic.

\section{b) Microscopic findings}

\section{1) Peripheral nervous system:}

The sciatic and sural nerves showed moderate to marked loss of myelinated fibers. In the former nerve, several large myelinated fibers remained whereas in the latter only fine fibers were seen. There was no evidence of myelin breakdown. 
The following cranial nerves were found to be within normal limits: III, IV, VI and motor V. In the optic nerve and tract, there was a mild pallor without definite loss of axons or gliosis. There was a slight depletion of myelinated fibers in the cranial sensory branch of the $\mathrm{V}$, the IX and $X$.

The posterior roots examined at various segmental levels showed a moderate to marked loss of myelinated fibers while the anterior roots appeared normal.

2) Central nervous system:

SPINAL CORD:

The spinal cord showed a marked loss of myelinated fibers in the posterior columns, the fasciculus cuneatus being slightly less involved. The crossed pyramidal and the spinocerebellar tracts were also markedly affected, the anterior spinocerebellar being less degenerated than the posterior fasciculus (fig. 1,2). There was a mild depletion of nerve fibers in the direct pyramidal tracts. The extent of the pyramidal tracts degeneration was similar at cervical and lumbar levels. In the Clarke's columns there was a moderate loss or neurons. Except for a slight loss of nerve cells in the cervical anterior horns, the motor neurons were preserved. The nerve cells of the intermediolateral columns were normal. The loss of neurons and myelinated nerve fibers was associated with a variable increase of astrocytes and/or glial fibers. There was no evidence of active myelin breakdown.

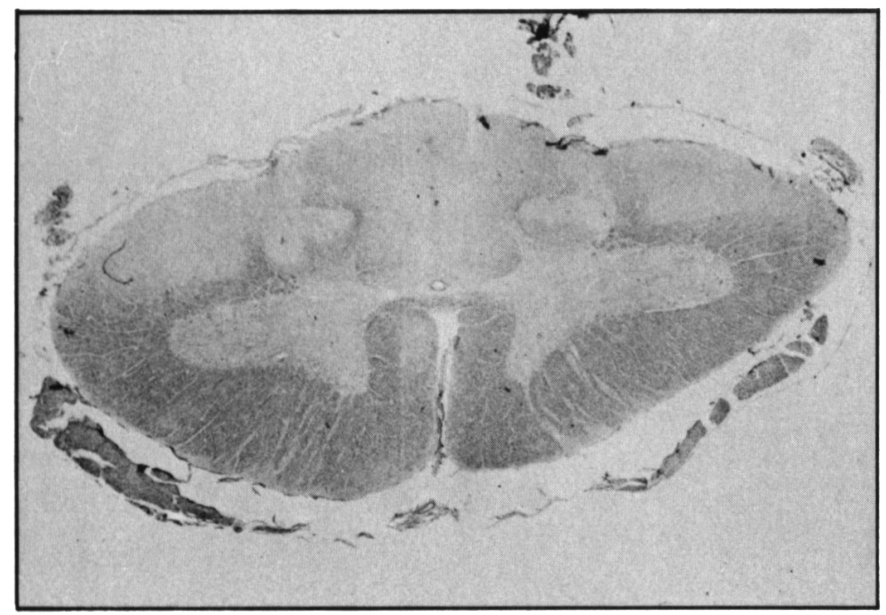

Figure I (Case 1)-Cervical spinal cord showing marked loss of myelinated nerve fibers in the posterior columns, the spinocerebellar tracts and the crossed pyramidal tracts. The fasciculus cuneatus is slightly less affected than the gracilis. The direct pyramidal tract shows slight degeneration. Luxol fast blue X 14 .

\section{BRAIN STEM:}

The degeneration of the pyramidal tract could be traced easily to the level of the medulla and pons where it was still moderate. The cerebral peduncle was however normally myelinated. There was a slight degeneration of the restiform body and the anterior portion of the median lemniscus. The superior cerebellar peduncle and the tractus solitarius were moderately degenerated.

There was a moderate loss of neurons in the gracile, cuneate and vestibular nuclei and in the inferior colliculus with an astrocytic proliferation. In these nuclei, most neurons appeared shrunken. The neurons of the inferior olivary and cochlear nuclei, the substantia nigra and the pons were normal.

\section{CEREBELLUM:}

There was a slight to moderate loss of Purkinje cells, particularly in the vermis, with a proliferation of astrocytes. Some Purkinje cells exhibited bizarre shapes and were misaligned. Several fusiform swellings of their axons (torpedoes) were observed in the upper granular layer (fig. 3). Along the parallel fibers of the molecular layer, a small number of swollen axons were also identified (fig. 4). There was a moderate loss of myelinated fibers in the white matter of the folia particularly in the vermis and in the hilum of the dentate nucleus (fig. 5). There was a

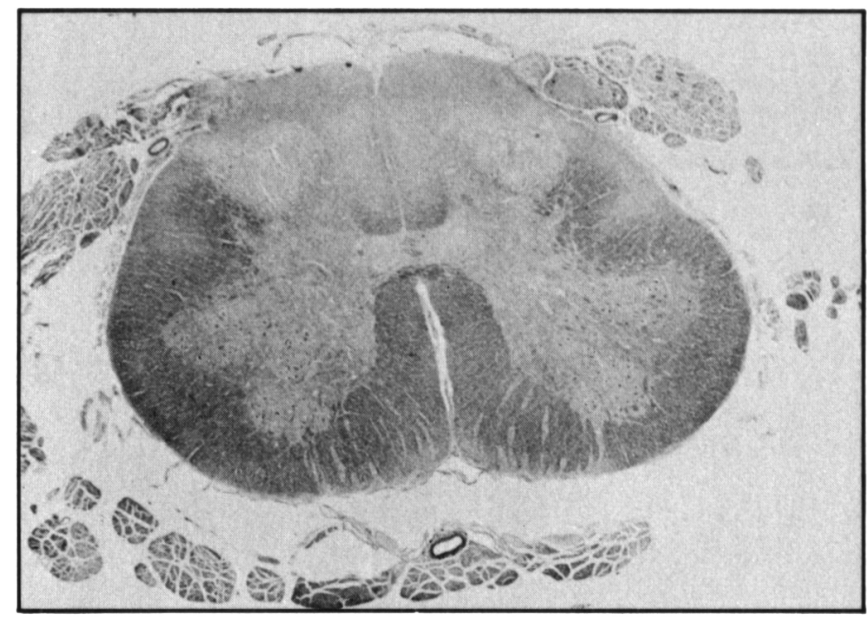

Figure 2 (Case 1) - Lumbar spinal cord showing marked loss of myelinated fibers in the fasciculi graciles, the spinocerebellar tracts and the crossed pyramidal tracts. Luxol fast blue X 14 .

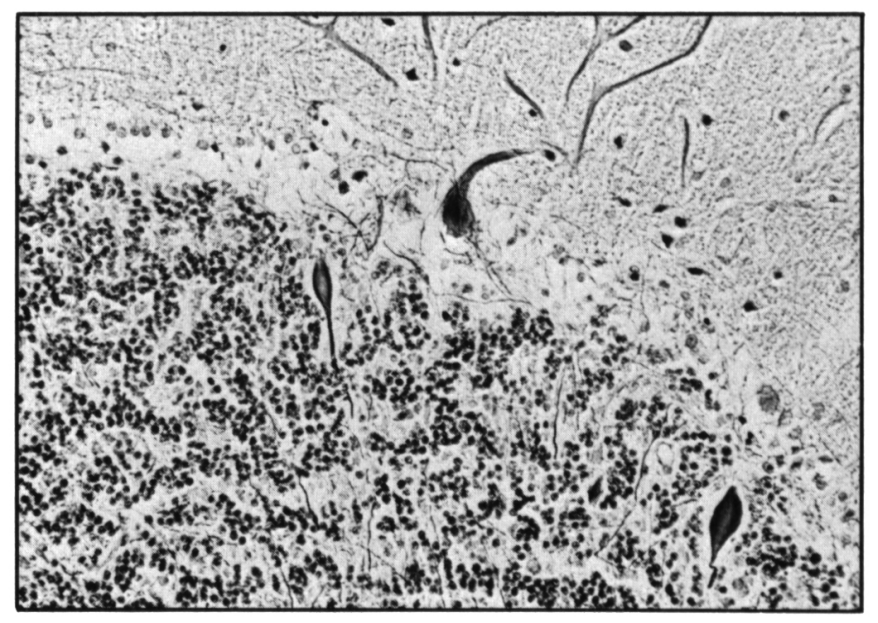

Figure 3 (Case 1) - Cerebellar cortex showing a loss of Purkinje cells. Note the atrophic neuron and the axonal swellings (torpedoes) in the superficial granular layer. Bodian X 212 .

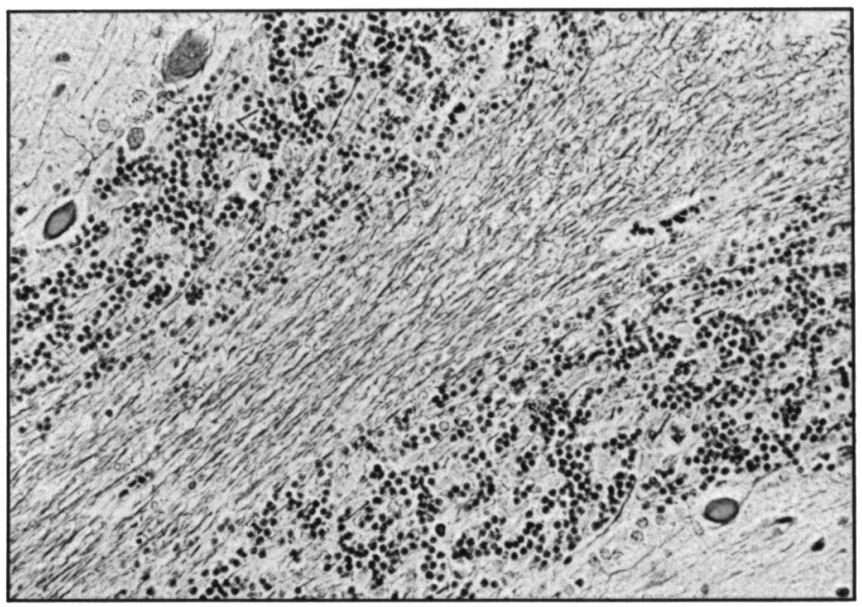

Figure 4 (Case 1) - Cerebellar cortex showing two axonal swellings along parallel nerve fibers of the lower molecular layer. Bodian X 212. 
moderate depletion of dentate neurons, most remaining nerve cells being shrunken.

\section{CEREBRUM}

The cerebral cortex, cerebral white matter and the internal capsule were normal. The subthalamic nucleus, the pallidum and the hypothalamus were also within normal limits. In the thalamus, there was a slight to moderate loss of neurons.

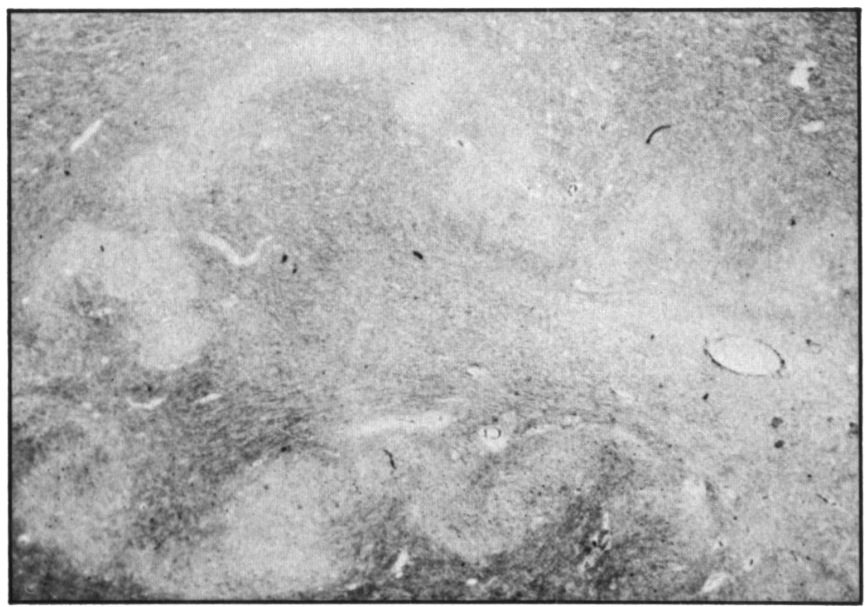

Figure 5 (Case I) - Dentate nucleus of cerebellum showing moderate loss of myelinated fibers in the hilum. Luxol fast blue X 13

\section{CASE 2 (M.T.-A-206,81) (Family Tree II)}

\section{Clinical Summary:}

This was a 12 year-old white female, product of a normal pregnancy and delivery with normal early development. She walked at the age of 10 months. At the age of 8 years, she was evaluated for progressive, but rapid gait ataxia with incoordination of upper extremities followed by transient improvement over the next six months. One year later, neurological examination revealed a limitation of convergence of the right eye, mild bilateral nystagmus, absence of deep tendon reflexes and a mild weakness of bilateral dorsiflexors with extensor plantar responses. A slight dysarthria and mild posterior column signs were present. There was limb, trunk and gait ataxia which progressed over the following 2 years to a point of needing help and support to walk. CT-scan, electroencephalography and electromyography were normal. However, there was absence of sensory nerve conduction velocity. She exhibited evidence of autonomic involvement with labile tachycardia and orthostatic hypotension. A discrete heart murmur was heard. The echocardiogram revealed a moderate concentric left ventricular hypertrophy. In view of her rapidly progressive scoliosis (from 240 to 450 in less than one year), she was admitted at the age of 12 for an elective corrective surgery. Unfortunately, she died 3 days after surgery from a fulminating beta-streptoccic septicemia, manifestated by metabolic acidosis, hyponatremia and septic shock.

An autopsy was carried out within 6 hours after her death.

\section{Summary of General Autopsy Findings:}

Pertinent findings outside the nervous system included bilateral pes cavus, scoliosis, slight muscular atrophy of the lower limbs and hypertrophic cardiomyopathy.

There was a status post recent Harrington procedure. In the respiratory system, there was massive bilateral pulmonary edema with intraalveolar hemorrages, bilateral pleural effusion and bilateral fibrinopurulent pleuritis with microabscesses in the right paravertebral muscles.

There were shock kidneys, generalized petechiae and hemorrages associated with recent thrombi in the colic mucosa. Chronic and acute passive congestion of liver and Hashimoto's thyroiditis were also found.

\section{Neuropathology:}

\section{a) Gross findings}

The brain weighed $1525 \mathrm{~g}$ in the fresh state and no abnormality was noted on external examination and after coronal sections.

Examination of cord showed a grey discoloration of the posterior columns and atrophic posterior roots.

\section{b) Microscopic findings}

1) Peripheral nervous system:

The following cranial nerves were found to be normal: III, IV, motor V, VI, VII.

A moderate to marked loss of myelinated fibers was observed in the VIII, IX, X, sensory V and in the posterior roots. A mild myelin pallor of the optic nerve withoug astrocytic reaction was noted. A marked loss of large myelinated fibers was noted in the posterior roots (fig. 6), the anterior being normal (fig. 7). In the posterior roots, numerous small myelinated fibers were seen. Several fibers were surrounded by a myelin sheath disproportionally too thin for the diameter of their axons. A spinal ganglion taken at $\mathrm{D}_{12}$ and the Gasserian ganglion exhibited a moderate loss of axons and myelin sheaths and a slight depletion of neurones. There was proliferation of capsule cells associated with

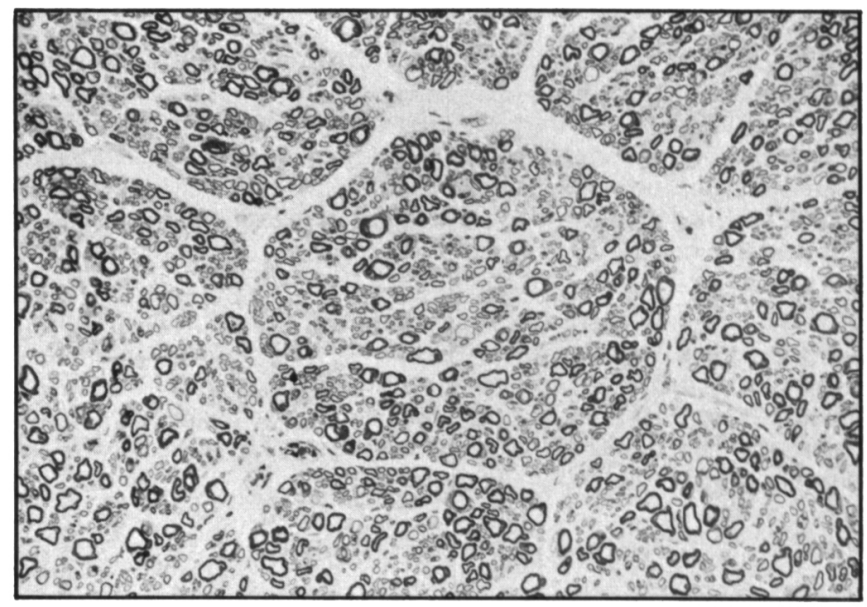

Figure 6 (Case 2) - Cross section of posterior cervical root fascicles showing marked loss of large myelinated nerve fibers. Note the unusual large number of small myelinated fibers. Several fibers contain thin myelin sheaths in relation to their axonal diameter. Semithin section stained with toluidine blue X 212 .

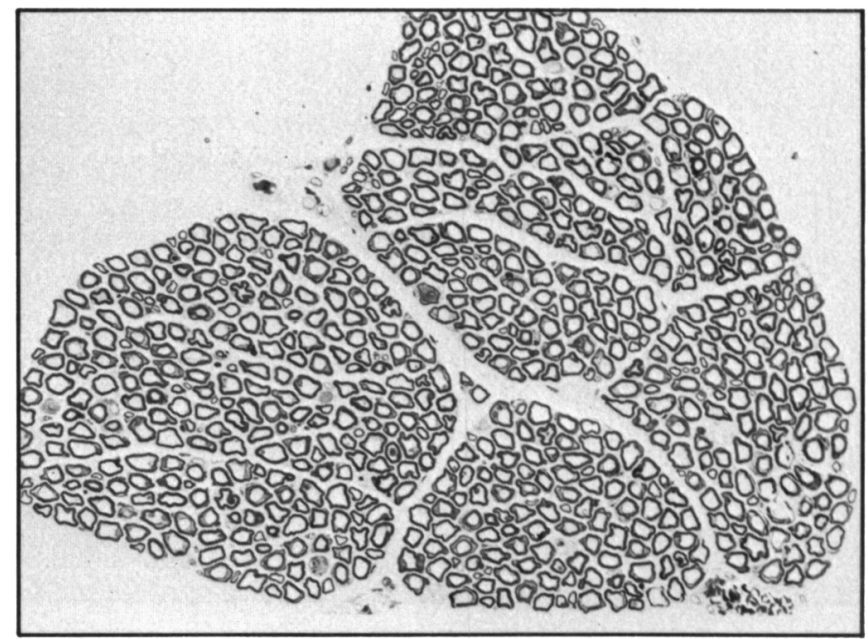

Figure 7(Case 2) - Cross section of anterior cervical root fascicles showing normal population of large and small myelinated nerve fibers. Semithin section stained with toluidine blue $X 212$. 
granular degenerative changes and/or swellings of some ganglion cells. A small number of nodules of Nageotte were noted. Focal swellings of perineuronal axons were frequently seen.

2) Central nervous system:

\section{SPINAL CORD:}

There was a marked loss of myelinated fibers in the fasciculus gracilis and cuneatus, the cuneatus being less affected. A moderate degeneration of the crossed and uncrossed pyramidal tracts, the anterior and posterior spinocerebellar tracts was also seen (fig. 8,9). There was no evidence of myelin breakdown. The neurons of the anterior horns and intermediolateral columns appeared normal.

\section{BRAIN STEM:}

The degeneration of the pyramidal tract was traceable to the medulla. There was also a slight degeneration of the restiform body, the superior cerebellar peduncle, the anterior portion of the median lemniscus and the descending tract of the $\mathrm{V}$. The tractus solitarius showed a moderate loss of myelinated fibers. In the nucleus gracilis and cuneatus, there was a slight increase in astrocytes with no clear cut loss of neurons. There were however numerous shrunken neurons with occasional axonal swellings. Shrunken neurons were also seen in the red nucleus where there was a moderate proliferation of astrocytes. The inferior olivary and pontine nuclei were normal.

\section{CEREBELLUM}

There was a loss of myelinated fibers with proliferation of astrocytes in the white matter of the folia especially in the vermis. A slight to moderate forcal loss of Purkinje cells was observed with several that remained showing acute ischemic changes. Several swollen axons were noted in the upper granular and in the molecular layer along parallel fibers. A moderate to marked loss of neurons was observed in the dentate nucleus.

\section{CEREBRUM:}

In the thalamus, there was a slight to moderate focal loss of neurons associated with a proliferation of astrocytes. Shrunken neurons with slightly eosinophilic cytoplasm suggesting acute anoxic changes were frequently seen in the thalamus, the striatum, the pallidum, the subthalamic nucleus and the claustrum. No significant changes were observed in the cerebral cortex and the hypothalamus.

\section{CASE 3 (M.L. 7737-83) (Family Tree V)}

\section{Clinical Summary:}

This patient was a 31 year-old female with classical Friedreich's ataxia. Her family history was unremarkable except for the presence of a younger sister with the same disease. The onset of a progressive ataxia was noted at the age of 7 with a rapid progressive dysarthria. She

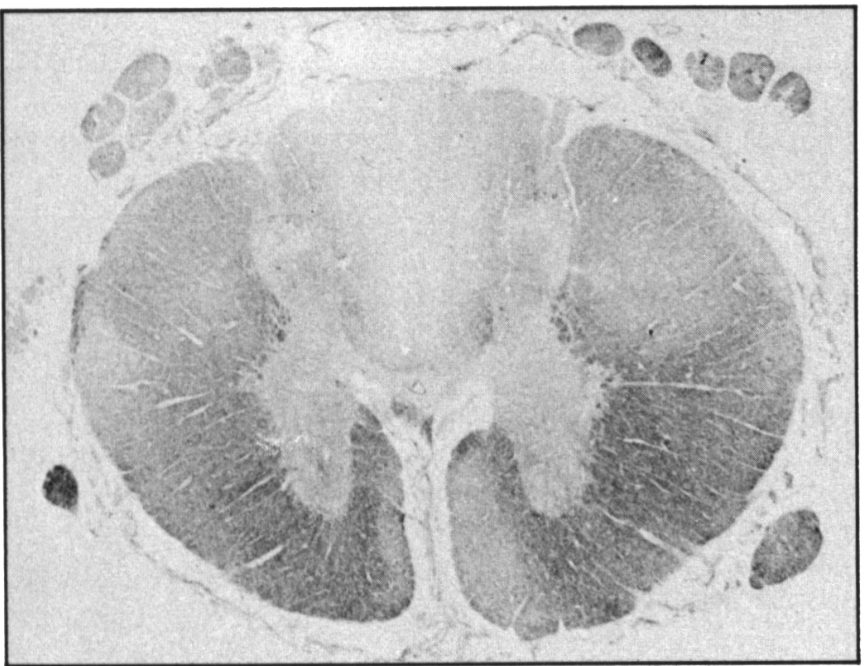

Figure 8(Case 2) - Cervical spinal cord showing marked loss of myelinated nerve fibers in the fasciculi graciles. The degeneration is moderate in the fasciculi cuneati, the spinocerebellar tracts and the pyramidal tracts (crossed and direct). Luxol fast blue X 14 was confined to a wheelchair at puberty. At the age of 22, neurological assessment showed a marked weakness of the lower extremities, a head, trunk and limb ataxia. Deep tendon reflexes were absent and bilateral extensive plantar responses were noted. A mild bilateral nystagmus, a $30^{\circ}$ dorsal kypho-scoliosis and a bilateral pes cavus were present. She exhibited moderate posterior columns signs. The echocardiogram did not reveal a cardiomyopathy but a small mitral valve prolapse. Clinical and laboratory data were reported by Geoffroy et al., 1976 (M.B.A.10). Over the years, her condition worsened to the point that she was unable to hold her head without support. She died of cardio-respiratory failure at the age of 31 .

An autopsy was performed within 8 hours of her death.

\section{Summary of General Autopsy Findings:}

Pertinent findings outside the nervous system included bilateral pes cavus, scoliosis and hypertrophic cardiomyopathy.

There were bronchopneumonia and chronic passive congestion with centrolobular necrosis of the liver.

\section{Neuropathology:}

\section{a) Gross findings}

The brain weight was not available. No significant changes were observed on external examination and after coronal sections. The available segments of spinal cord were moderately atrophic and the posterior roots were thin and greyish.

\section{b) Microscopic findings}

1) Peripheral nervous system:

No peripheral nerve was available for examination. In the posterior roots, there was a marked loss of myelinated fibers. The anterior roots appeared normal. In a thoracic spinal ganglion, there was a slight neuronal loss associated with some nodules of Nageotte. There was a proliferation of capsule cells around some degenerating neurons and a few perineuronal axonal swellings were identified.

2) Central nervous system:

\section{SPINAL CORD:}

A marked loss of myelinated fibers was observed in the fasciculus gracilis and cuneatus, the pyramidal tracts and the spinocerebellar tracts, the anterior being less affected than the posterior (Fig. 10). In addition, there was a moderate loss of neurons with atrophy of the remaining ones in the Clarke's columns. The neurons of the anterior horn were normal. There was no evidence of active breakdown of myelin.

\section{BRAIN STEM:}

The pyramidal tracts degeneration was traceable to the pons. In the restiform body, the inferior cerebellar peduncle and the anterior por-

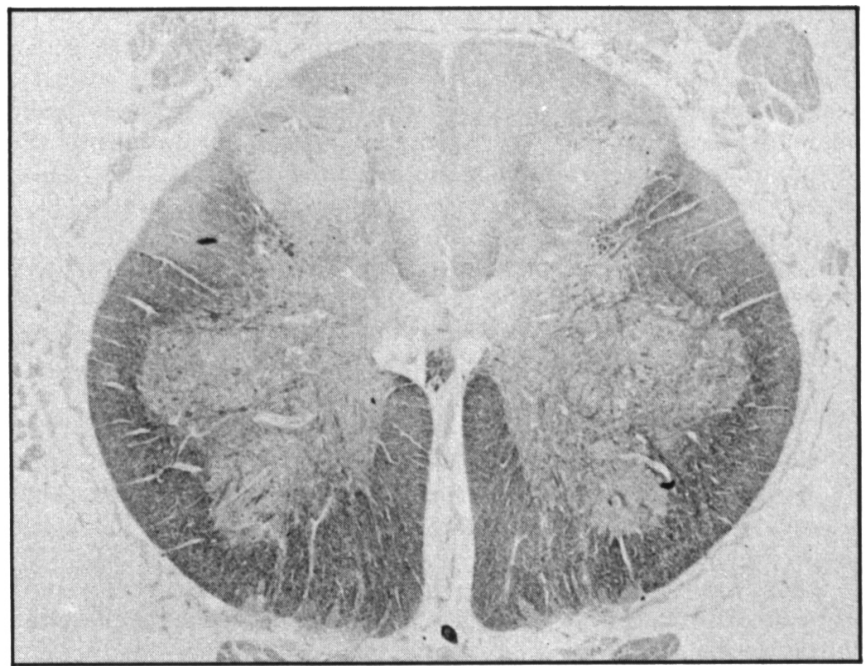

Figure 9 (Case 2) - Lumbar spinal cord showing marked loss of myelinated nerve fibers in the fasciculi graciles. The degeneration is moderate in the posterior spinocerebellar tracts and the crossed pyramidal tracts and absent in the uncrossed pyramidal tracts. Luxol fast blue X 14 
tion of the medullary median lemniscus, there was a slight myelin pallor. A moderate loss of neurons was found in the gracile, cuneate and accessory cuneate nuclei. The neurons of the sensory $V$ were shrunken.

CEREBELLUM:

The cerebellar white matter was normal except for a moderate loss of myelinated nerve fibers in the hilum of the dentate nucleus. A slight focal loss of Purkinje cells was observed together with a small number of axonal swellings (torpedoes).

CEREBRUM:

The cerebral cortex and white matter appeared normal. There was a slight focal neuronal loss in the thalamus and pallidum.

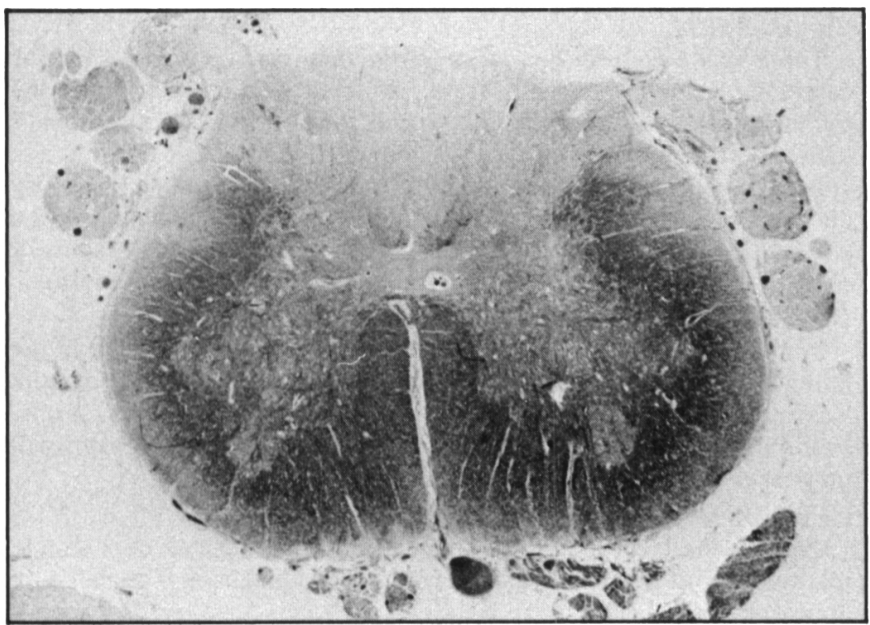

Figure 10(Case 3) - Lumbar spinal cord showing marked loss of myelinated nerve fibers in the fasciculi graciles, the spinocerebellar tracts and the crossed pyramidal tracts.

\section{CASE 4 (G.H.-A-257-76) (Family Tree IV)}

\section{Clinical Summary:}

This was a 28 year-old male suffering from Friedreich's ataxia since the age of 5 . He became confined to a wheelchair at the age of 13. A neurological investigation was carried out at the age of 21 (data published by Geoffroy et al., 1976 S.B.L. 10). At that time, he showed a marked dysarthria, cerebellar ataxia and posterior columns deficit. A mild mixed peripheral neuropathy with normal cerebro-spinal fluid protein was demonstrated. A moderate dorsolumbar scoliosis and a pes cavus were present. No nystagmus was recorded except over the following 6 months preceding his death. Cardiac manifestations were detailed in a previous publication (case 3 , Lamarche et al., 1980). He died at the age of 28 after an episode of acute abdominal manifestations.

An autopsy was carried out less than $\mathbf{4}$ hours after his death.

\section{Summary of General Autopsy Findings:}

Pertinent findings outside the nervous system included bilateral pes cavus, scoliosis, muscular atrophy affecting the lower extremities and hypertrophic cardiomyopathy.

There was a granulomatous process compatible with sarcoidosis in the lungs, the spleen, the lymph nodes, the liver and the heart. Erosive gastritis was also observed.

\section{Neuropathology:}

\section{a) Gross finding}

The brain weighed $1480 \mathrm{~g}$ in the fresh state. No gross lesions were seen on external examination and after coronal sections. The spinal cord was markedly atrophic.

\section{b) Microscopic findings}

1) Peripheral nervous system:

The sural nerve showed a marked loss of large myelinated fibers. The sciatic nerves displayed a loss of nerve fibers but several large myelinated axons were found.
The following cranial nerves appeared normal: II, III, IV, VI, VII and XII. A moderate to marked loss of myelinated fibers was observed in the IX and V sensory nerves. The posterior roots at all levels exhibited moderate to marked loss of myelinated fibers. The corresponding anterior roots appeared normal.

The spinal ganglia displayed a marked loss of myelinated fibers and nerve cells with an intensive proliferation of capsule cells, numerous nodules of Nageotte being identified (Fig. 11). Several perineuronal axonal swellings were noted (Fig. 12). Some ganglion cells showed a granular degeneration.

2) Central nervous system:

SPINAL CORD:

There was a marked loss of myelinated nerve fibers in the posterior columns, the fasciculus gracilis being more affected than the fasciculus cuneatus. In the crossed pyramidal and spinocerebellar tracts, the degeneration was moderate (Fig. 13). A minimal loss of nerve fibers was observed in the direct pyramidal tracts. A marked deletion of neurons was seen in the Clark's columns with shrinkage of the remaining ones. The neurons of the intermediolateral columns and the anterior horns appeared normal. There was no evidence of active breakdown of myelin.

BRAIN STEM:

A slight degeneration was noted in the restiform body, the superior cerebellar pedundle, the spinal tract of $\mathrm{V}$, the fasciculus solitarius, the

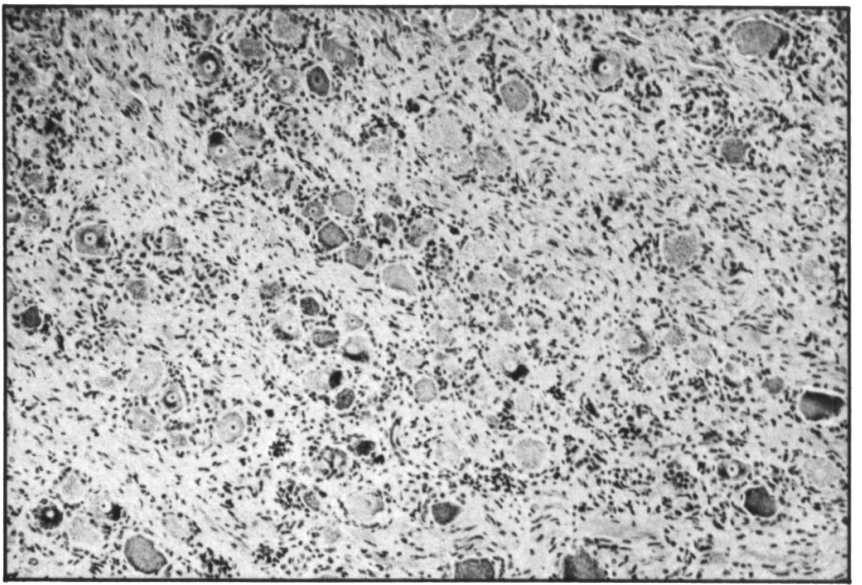

Figure II (Case 4) - Thoracic spinal ganglion showing marked loss of nerve cells, proliferation of capsular cells and several nodules of Nageotte. Luxol fast blue $X 84$

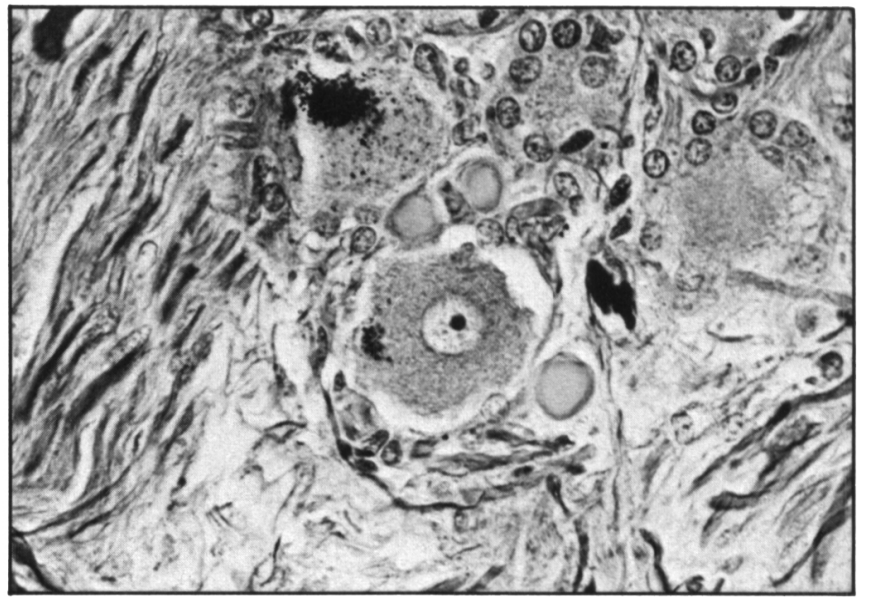

Figure 12 (Case 4) - Thoracic spinal ganglion showing multifocal perineuronal axonal swellings. The ganglion cell appears well preserved. Bodian $X$ 530 . 
pontine and medullary pyramidal tract and the medullary median lemniscus. A moderate loss of neurons with an increase in astrocytes was noted in the vestibular and cochlear nuclei, the sensory nucleus of $\mathrm{V}$, the red nucleus and the inferior colliculus. Most of the remaining neurons in these nuclei were shrunken. The inferior olivary nuclei, and the substantia nigra appeared normal.

\section{CEREBELLUM}

In the cerebellar cortex, there was a generally slight loss of Purkinje cells with proliferation of astrocytes. Several axonal swellings (torpedoes) were noted in the upper granular layer. The cerebellar white matter was normal except in the hilum of the dentate nucleus where a moderate loss of myelinated fibers was noted. This was associated with a marked loss of dentate neurons with shrinkage of the remaining ones.

\section{CEREBRUM:}

The cerebral cortex including the motor area and the cerebral white matter were normal.

In the thalamus, there was a slight to marked focal loss of neurons and in the mamillary body, a slight neuronal depletion. The subthalamic nucleus appeared normal.

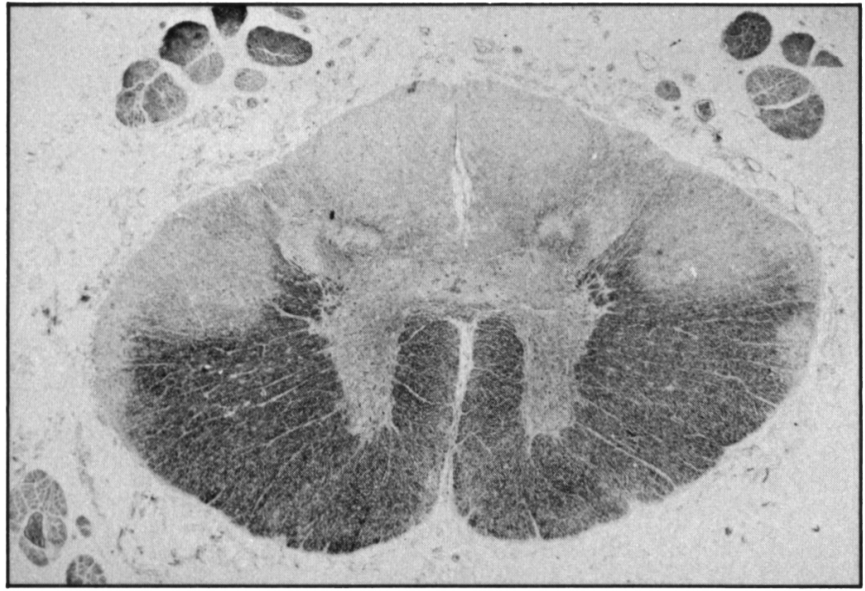

Figure 13 (Case 4) - Thoracic spinal cord showing marked degeneration of the fasciculi graciles, the spinocerebellar tracts and the crossed pyramidal tracts. Luxol fast blue $X 13$

\section{CASE 5 (R.T.-A-25-72) (Family Tree I)}

\section{Clinical Summary:}

This was a 22 year-old male suffering from Friedreich's ataxia since the age of 9 and confined to a wheel chair since the age of 12. Two brothers affected with the same disease died, the eldest at the age of 25 and the youngest (D.T.) at the age of 21 (case No. 6). Two sisters with classical Friedreich's ataxia are still alive. Pregnancy and delivery were normal. Growth and development were normal up to the age of 5 when he was examined for difficulties in walking. Scoliosis was noted at the age of 14. Cardiac manifestations were first noted around the age of 18 . Detailed clinical data are reported in a previous publication (case 2 Lamarche et al., 1980). He died at the age of 21 after a progressive cardio-respiratory insufficiency, complicated by a persistent state of shock and anuria.

An autopsy was carried out 3 hours after death.

\section{Summary of General Autopsy Findings:}

Pertinent findings outside the nervous system included bilateral pes cavus, scoliosis and cardiomyopathy.

In the lungs, there was a marked chronic passive congestion with intraalveolar edema and hemorrages. An early bronchopneumonia was found in the lower lobes. Marked chronic passive congestion of liver was also seen.

\section{Neuropathology:}

\section{a) Gross findings}

The brain weighed $1310 \mathrm{~g}$ and no gross lesion was observed on external examination or after coronal sections. The spinal cord was described as atrophic.

\section{b) Microscopic findings}

1) Peripheral nervous system:

No spinal ganglia or peripheral nerve was available for study. There was a moderate loss of myelinated nerve fibers in the posterior roots, the anterior roots appearing normal. A slight myelin pallor of the optic tracts without astrocytic reaction was noted

2) Central nervous system:

\section{SPINAL CORD:}

There was a marked loss of myelinated fibers in the fasciculus gracilis and cuneatus, the spinocerebellar and pyramidal tracts being moderately involved (Fig. 14). The anterior horns were normal except for rare chromatolytic neurons. In the Clarke's columns, there was a slight loss of neurons with shrinkage of the remaining ones.

\section{BRAIN STEM:}

There was a slight degeneration of the restiform body, the superior cerebellar peduncle being moderately affected. The degeneration of the pyramidal tract was traceable to the pons. There was a moderate loss of neurons with shrinkage of the remaining ones in the nucleus gracilis, cuneatus, accessory cuneatus, red nucleus, vestibular nuclei, sensory nucleus of the V, subthalamic nucleus and inferior colliculus. Swollen axons were identified in the nucleus gracilis, cuneatus and the sensory of the $\mathrm{V}$. The substantia nigra and the nuclei pontis appeared normal.

\section{CEREBELLUM:}

The white matter of the cerebellum appeared normal except in the hilum of the dentate nucleus where a moderate loss of myelinated fibers was seen. This was associated with a marked loss of dentate neurons. In the cortex, there was a slight focal loss of Purkinje cells and a small number of swollen axons (torpedoes) were found.

CEREBRUM:

The cerebral cortex and white matter were normal. A slight to marked loss of neurons was detected in the thalamus. The pallidum appeared normal.

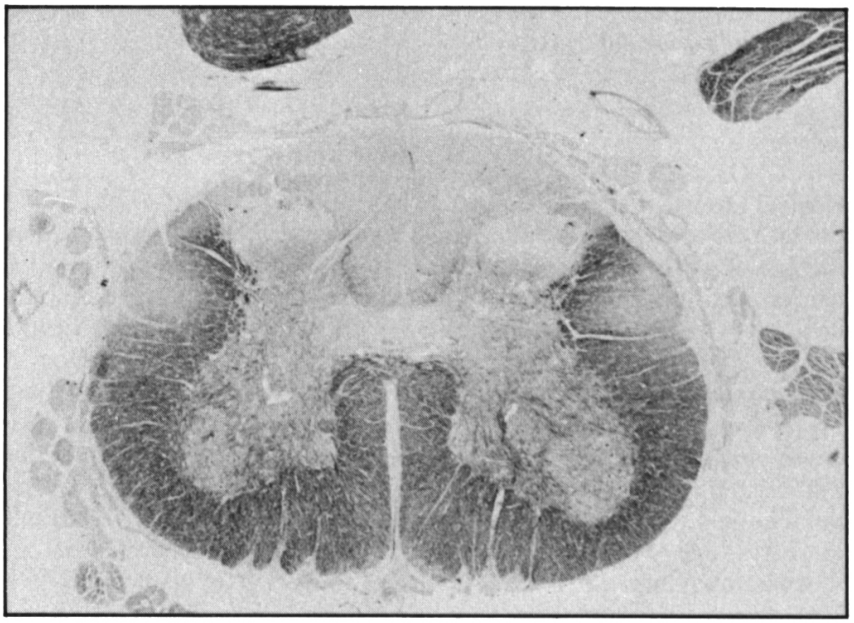

Figure 14 (Case 5) - Lumbar spinal cord showing marked loss of myelinated fibers in the fasciculi graciles, the spinocerebellar tracts and the crossed pyramidal tracts. Luxol fast blue X 14.

\section{CASE 6: (D.T.-A-223-80) (Family Tree I)}

\section{Clinical Summary:}

This was a 21 year-old male, product of a normal pregnancy, birth and early development, except a delay in walking at the age of 3. Around the age of 5, a progressive steady gait disturbance appeared. At the age of 13 , he was confined to a wheel chair and dysarthria was observed. At the age of 17 , a neurological evaluation was carried out and previously reported as S.B.L.-16 Geoffroy et al. (1976). Pertinent findings included a marked spino-cerebellar ataxia, a moderate posterior columns deficit and bilateral extensor plantar responses. A mild nystagmus was present. A $77^{\circ}$ scoliosis with moderate rotation and a bilateral pes cavus was observed. A pulmonary restrictive syndrome 
and a left ventricular asymetric hypertrophic cardiomyopathy were demonstrated. At the age of 21 , he underwent a corrective operation for scoliosis. One year prior to his death, he was admitted to hospital for a drug intoxication and an arterial embolus of the right foot. Two weeks prior to his death, he refused to take all medication prescribed at the previous admission. A few days before his death, he presented recurrent vomiting and hypersomnolence. He was admitted to a local hospital for uncontrolled vomiting and died after a few hours of coma.

An autopsy was carried out within 6 hours.

\section{Summary of General Autopsy Findings:}

Pertinent findings outside the nervous system included bilateral pes cavus, scoliosis, muscular atrophy of the lower limbs and hypertrophic cardiomyopathy associated with mural thrombi in both auricles and the right ventricle.

There was acute and chronic passive congestion of the liver with centrolobular necrosis and multiple old infarcts were observed in the kidneys.

\section{Neuropathology:}

\section{a) Gross findings}

The brain weighed $1240 \mathrm{~g}$ in the fresh state. External examination revealed several subacute infarcts in the cerebellum especially in the vermis and the inferior portion of the hemispheres. Coronal sections showed numerous small subacute infarcts disseminated in the cerebral cortex, the largest being localized in the temporo-parieto-insular region in the distribution of the middle cerebral artery. Several similar lesions were detected in the cerebellar cortex. The spinal cord was markedly atrophic and the posterior roots were gray and atrophic.

\section{b) Microscopic findings}

1) Peripheral nervous system:

The sural nerve showed a marked depletion of myelinated fibers particularly of the large ones (Fig. 15). The posterior roots showed similar changes at all levels. The anterior roots were normal.

No significant changes were seen in the cranial nerves I, III, IV. The optic nerve and tract showed a moderate pallor of the myelinated fibers with only a slight increase in astrocytes.

A section of lumbar spinal ganglion showed a marked loss of myelinated fibers and a moderate neuronal depopulation associated with a proliferation of satellite cells and several nodules of Nageotte. Numerous nerve cells were vacuolated, others showed a granular degeneration. Several axonal swellings were identified. A thoracic sympathetic ganglion was found to be normal.

2) Central nervous system:

\section{SPINAL CORD:}

A marked loss of myelinated fibers was found in the posterior columns, the fasciculus gracilis being more affected than the cuneatus. There was a moderate degeneration of the pyramidal and spinocerebellar tracts, the anterior tracts being less involved than the posterior. The direct pyramidal tract was normal. There was a marked loss of neurons in the Clarke's columns with shrinkage of the remaining nerve cells. The neurons of the intermediolateral were normal. Occasional chromatolytic nerve cells were found in the anterior horns which were otherwise normal.

BRAIN STEM:

The pyramidal degeneration was traceable to the medulla and pons. There was a degeneration of the restiform body and the superior cerebellar peduncles, the latter structure being moderately affected. A slight degeneration was also noted in the median lemniscus and the entering trigeminal root.

There was a slight to moderate loss of neurons in the vestibular nucle with corresponding increase in astrocytes, the remaining neurons being shrunken. The following nuclei were found to be normal: substantia nigra, nuclei pontis and inferior olivary nuclei. Marked acute anoxic changes were noted in the red nucleus.

\section{CEREBELLUM:}

Several small foci of liquefactive necrosis were observed in the cortex. In the other portions of the cortex, there was a slight loss of myelinated fibers. A slight focal loss of Purkinje cells was noted, several axonal swellings (torpedoes) being seen in the upper granular layer. In the dentate nucleus, there was a marked loss of neurons, with shrinkage of the remaining ones. The hilum of the dentate nucleus showed a moderate loss of myelinated fibers.

\section{CEREBRUM:}

Several areas of liquefactive necrosis were observed in the cerebral cortex and the thalamus. There was a slight to marked focal loss of neurons in the thalamus, in the subthalamic nucleus and in the pallidum with a proliferation of astrocytes. In these nuclei, a various number of neurons showed ischemic changes.

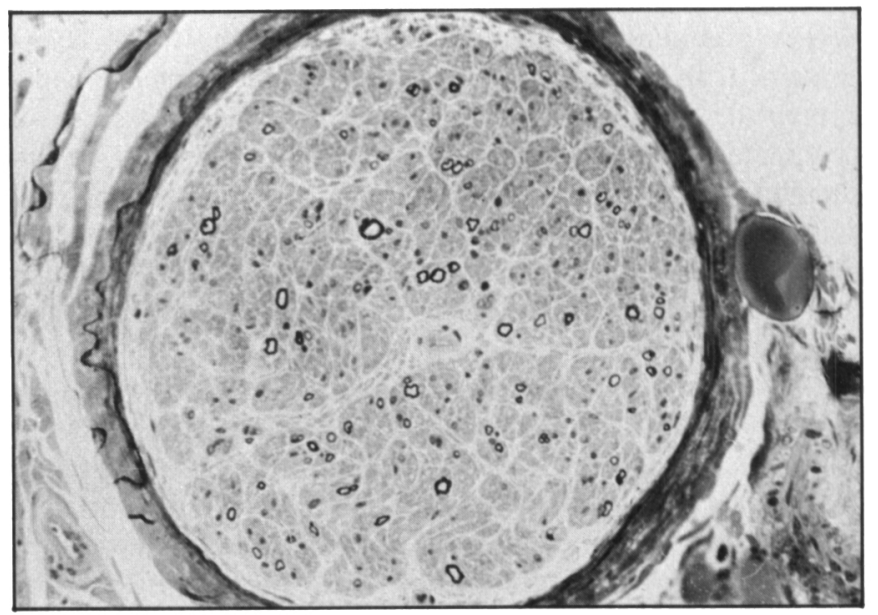

Figure 15 (Case 6) - Cross section of a fascicle from sural nerve showing severe loss of myelinated nerve fibers, particularly the large ones. Most of the remaining myelinated fibers are small. Semithin section stained with toluidine blue X 212 .

\section{Discussion}

All 6 cases met the clinical diagnostic criteria of Friedreich's ataxia as defined by the Quebec Cooperative study (Geoffroy et al., 1976). The essential clinical features included age of onset between 2 to 9 , progressive unremitting ataxia of gait, deep tendon areflexia and progressive muscle weakness in lower extremities, dysarthria and decreased vibration and position sense in lower limbs. All patients also had pes cavus, scoliosis, cardiomyopapthy and extensor plantar responses. It is worth mentioning that the youngest patient, age 12 at the time of death (case 2), showed a rapid progression of her gait disturbance but was not yet confined to a wheel-chair. Among the accessory signs and symptoms, the only one observed was nystagmus found in 5 of 6 cases, usually mild and occuring late during the course of the disease. None of the patients showed optic atrophy. None of these 6 cases had clinical diabetes mellitus and 3 of 4 patients investigated for chemical diabetes were found to be normal. On the basis of the uniform clinical picture and the clinical course, we can state that we are dealing with a homogeneous group of patients from five distinct families. In these patients, the cardiac dysfonctions and the scoliosis were contributing factors to their disability and death.

The anatomical lesions found in the peripheral and central nervous system were similar in all 6 cases and do not differ from those described in the literature in the usual form of Friedreich's ataxia (Hughes et al., 1968; Netsky, 1968; Oppenheimer, 1979). The clinical findings in all 6 cases correlate closely with the histological lesions found in the peripheral nervous system and the spinal cord. In case 2 , the extent and the severity of the spinal cord involvement after only 4 years of clinical evolution was surprising. This observation seems consistent with a rapid 
progression of the disease process although there was no evidence of myelin breakdown in the lesions. Except for occasional neurons showing chromatolysis in 2 cases, the anterior horns appeared normal. No lesions were detected in the anterior roots, the cranial motor nerve and nuclei and the intermediolateral columns of the spinal cord. The loss of large myelinated fibers in the posterior roots and the sensory cranial nerves was generally marked in all cases and at all levels examined. In our youngest case, age 12, the posterior roots contained, in addition, an unusually large number of small myelinated fibers together with several fibers with a myelin sheath too thin for their axonal diameter. These findings are consistent with a process of segmental demyelination and remyelination secondary to axonal degeneration as reported in the sural nerve of patients with Friedreich's ataxia by Dyck and Lais, 1973. Similarly, we have also found evidence of segmental demyelination and remyelination in a spinal ganglion biopsy from a 17 year old patient with Friedreich's ataxia (Lamarche et al., 1982). Our observations in the posterior roots and the spinal ganglion provide further support for a dying back process (Cavanagh, 1979) in Friedreich's ataxia, the axonal degeneration slowly progressing from the distal portion of the fiber toward its cell body.

Pathological changes in the brain stem were rather variable although slight to moderate myelin loss was constantly found in the restiform body, the superior cerebellar peduncles and the pyramidal tract up to the pons. Neuronal loss associated with proliferation of astrocytes was consistently observed in the nucleus gracilis and cuneatus. In other nuclei, the changes were variable and in some cases probably related to axonia. Neuronal loss with gliosis and shrunken neurons were observed in the vestibular nuclei in 4 cases, in the inferior colliculi in 3 , and in the red nucleus in 3 . The pallidum and the subthalamic nucleus were found to be affected in one case. None of the patients has shown clinical evidence of involvement of the vestibular and oculomotor pathways except nystagmus. Although clinical (Tyrer, 1975), electrophysiological (Monday et al., 1978; Kirkham et al., 1979) and pathological (Urich et al., 1957; Boudin et al., 1972; Oppenheimer, 1979) evidence of involvement of vestibular and oculomotor pathways has been shown in several patients with Friedreich's ataxia, correlations between the clinical findings and the anatomical lesions have been rarely made. This could be related partly in some instances to incomplete examination or minimal anatomical involvement at the time of examination or death. No conclusive clinicopathological correlations have been possible following the study of the vestibular pathways in our cases because of insufficient anatomical material.

In the cerebellum, the most striking alterations were a moderate to marked loss of dentate neurons and myelinated fibers in the hilum of the dentate nucleus and the presence of axonal swellings in the cortex. To our knowledge, axonal swellings in the cerebellar cortex have not been described in Friedreich's ataxis except in one observation (Urich et al., 1957). We have found fusiform swellings of Purkinje cells axons (torpedoes) in all 6 cases. In addition, axonal swellings were detected aiong parallel fibers of the molecular layer in 2 cases. Axonal "torpedoes" are usually found in the degenerative diseases when Purkinje cells undergo simple atrophy (Blackwood, 1976) and have been observed mainly in olivopontocerebellar atrophy (Koeffen and Barron, 1983) and in primary degeneration of the cerebellar granular layer (Netsky, 1968). The presence of axonal swellings in the granular and molecular layers suggests that both the Purkinje cells and the granules are involved in the degenerative process. Although there was only slight to moderate loss of Purkinje cells in our cases, this neuronal depletion could possibly be enough to explain, by trans-synaptic degeneration, the constant loss of nerve cells and nerve fibers observed in the dentate nucleus. Loss of Purkinje cells is fairly common in Friedreich's ataxia (Greenfield, 1954) and has been attributed to anoxia by some authors (Oppenheimer, 1973). This possibility cannot be ruled out. However, the finding of axonal swellings as the result of neuronal atrophy could provide an additional possible mechanism.

\section{REFERENCES}

Blackwood W (1976) Normal structure and general pathology of the nerve cell and neuroglia. In: Greenfield's Neuropathology p 1-42, 3rd ed. Edward Arnold, London.

Boudin G, Grossiord A, Guillard A, Mikol J, Lacert P (1972) Maladie de Friedreich avec atteinte systématisée sus-médullaire. Rev Neurol 127: $441-456$.

Cavanagh JB (1979) The "dying back" process. A common denominator in many naturally occurring and toxic neuropathies. Arch Path Lab Med, 103: 659-664.

Dyck PJ, Lais AC (1973) Evidence for segmental demyelination secondary to axonal degeneration in Friedreich's ataxia. In: Kakulas BA (ed); Clinical studies in myology. Excerpta Medica Amsterdam, 253-263.

Geoffroy G, Barbeau A, Breton G, Lemieux B, Aubé M, Léger C, Bouchard JP (1976) Clinical description and roentgenologic evaluation of patients with Friedreich's ataxia. Can J Neurol Sci 3: 279-286.

Greenfield JG (1954) The spinocerebellar degenerations. Blackwell Scientific Publications, Oxford.

Hughes JT, Brownell B, Hewer RL (1968) The peripheral sensory pathway in Friedreich's ataxia: an examination by light and electron microscopy of the posterior nerve roots, posterior root ganglia and peripheral sensory nerve in cases of Friedreich's ataxia. Brain 91: 803-820.

Kirkham TH, Guitton D, Katsarkas A, Kline LB, Andermann E (1979) Oculomotor abnormalities in Friedreich's ataxia. Can J Neurol Sci 6: $167-172$.

Koeffen AH, Barron KD Neuropathology of olivopontocerebellar atrophy In: Olivopontocerebellar atrophy. Duvoisin R, and Plaitakis A (eds) Raven Press New York 1983.

Lamarche JB, Coté M, Lemieux B (1980) The cardiomyopathy of Friedreich's ataxia. Morphological observations in 3 cases. Can J Neurol Sci 7: 389-396.

Lamarche J, Luneau C, Lemieux B (1982) Ultrastructural observations on spinal ganglion biopsy in Friedreich's ataxias. A preliminary report. Can J Neurol Sci 9: 137-139.

Monday LA, Lemieux B, St-Vincent H, Barbeau A (1978) Clinical and electronystagmographic findings in Friedreich's ataxis. Can J Neurol Sci 5: $71-73$.

Netsky MG (1968) Degenerations of the cerebellum and its pathway, In Minckler J (Ed). Pathology of the nervous system Vol 1: 1163-1185. McGraw-Hill Book Co. New York.

Oppenheimer DR (1979) Brain lesions in Friedreich's ataxia. Can J Neurol Sci 6: 173-176.

Tyrer J (1975) Friedreich's ataxia. In: Handbook of Clinical Neurology, vol 21: 319-364. (System disorders and atrophies Part I) Vinken PJ and Bruyn GW, (ed) North Holland Publishing Co., Amsterdam.

Urich H, Normand RM, Lloid OC (1957) Suprasegmental lesions in Friedreich's ataxia. Confin Neurol (Basel) 17: 360-371. 